\title{
Synthesis of monolayer $\mathrm{MoS}_{\mathbf{2}}$ by CVD approach \\ Y. Y. WEN, X. B. ZENG*, X. X. CHEN, W. Z. WANG, J. DING , S. E. XU \\ School of Optical and Electronic Information, Huazhong University of Science and Technology, Wuhan 430074, China
}

*Corresponding author: eexbzeng@163.com

Keywords: CVD, monolayer $\mathrm{MoS}_{2}$, Raman, photoluminescence, bandgap

\begin{abstract}
The monolayer $\mathrm{MoS}_{2}$ thin film was deposited directly on quartz substrate by chemical vapor deposition (CVD) approach using $\mathrm{MoO}_{3}$ and sulfur powders as reactants. Raman spectroscopic and photoluminescence (PL) spectroscopic analyses were conducted to evaluate the structural and optical property of the grown $\mathrm{MoS}_{2}$ thin films. The Raman characteristic peaks in $385 \mathrm{~cm}^{-1}\left(\mathrm{E}_{2 \mathrm{~g}}{ }^{1}\right)$ and $405 \mathrm{~cm}^{-1}\left(\mathrm{~A}_{1 \mathrm{~g}}\right)$ prove the grown film is monolayer $\mathrm{MoS}_{2}$. In comparision, we also found in some areas of the grown fim is bulk $\mathrm{MoS}_{2}$ film, the Raman characteristic peaks of which are $384 \mathrm{~cm}^{-1}\left(\mathrm{E}_{2 \mathrm{~g}}{ }^{1}\right)$ and $409 \mathrm{~cm}^{-1}\left(\mathrm{~A}_{1 \mathrm{~g}}\right)$. And two pronounced emission peaks at 620 and $670 \mathrm{~nm}$ were observed in photoluminescence spectrum of monolayer $\mathrm{MoS}_{2}$. The results suggest that we synthesize monolayer $\mathrm{MoS}_{2}$ with optical bandgap of $1.85 \mathrm{eV}$.
\end{abstract}

\section{Introduction}

Recently, researchers have been focusing on graphene-like 2D materials, aiming at overcoming the shortage of graphene and broadening its range of applications. Monolayer molybdenum disulphide $\left(\mathrm{MoS}_{2}\right)$ is a typical example from the layered transition-metal dichalcogenide (TMD) family of materials with a direct bandgap of $1.8 \mathrm{eV}^{[1]}$. Due to its attractive electronic, optoelectronic, and mechanical properties ${ }^{[2-5]}$, great effort has been devoted to the study of $\mathrm{MoS}_{2}$. Bulk $\mathrm{MoS}_{2}$ is known to have an indirect bandgap of $\sim 1.2 \mathrm{eV}$ and widely used as lubricants at first, whereas single-layer $\mathrm{MoS}_{2}$ is a direct gap semiconductor ${ }^{[1,5,6]}$ with a bandgap of $1.8 \mathrm{eV}$. The direct bandgap also results in photoluminescence from monolayer $\mathrm{MoS}_{2}$, which opens the possibility of many optoelectronic applications ${ }^{[7]}$. In addition, bulk (or multilayer) $\mathrm{MoS}_{2}$ exhibits relatively high in-plane carrier mobility comparable to that of crystalline silicon ${ }^{[7]}$ as well as robust mechanical and chemical properties, which makes it an attractive material for making flexible electronic devices with high performance and long lifetime $e^{[3,8,9]}$.

The current approaches for fabricating thin layers of $\mathrm{MoS}_{2}$ are as follows, mechanical and liquid exfoliation ${ }^{[10,11]}$ of bulk materials, physical vapor deposition ${ }^{[12]}$ and chemical vapor deposition ${ }^{[13 \text {, }}$ ${ }^{14]}$. CVD is a direct bottom-up method for producing large-area uniform poly crystalline thin films. In this article, we describe the approach for preparing monolayer $\mathrm{MoS}_{2}$ films on quartz by CVD. We used $\mathrm{MoO}_{3}$ powder and sulfur powder is as reactants. Furthermore, structural, spectral, and morphology of the monolayer $\mathrm{MoS}_{2}$ films were studied.

\section{Experimental Procedure}

The quartz substrate was treated according to the standard Radio Corporation of America (RCA) cleaning procedure. As illustrated in Figure 1, monolayer $\mathrm{MoS}_{2}$ film was synthesized in a horizontal quartz tube furnace with sulfur and $\mathrm{MoO}_{3}$ powders as reactants. The $\mathrm{MoO}_{3}(0.1 \mathrm{~g}, 99.99 \%)$ was placed downstream in a ceramic boat and loaded into the central uniform-temperature zone of the furnace. The quartz substrate was faced down and mounted on the top of boat. Another ceramic boat with sulfur powder $(0.8 \mathrm{~g}, 99.9 \%)$ was placed upstream in a low-temperature zone. The center of the furnace was heated from room temperature to $550^{\circ} \mathrm{C}$ in $30 \mathrm{~min}$ at a rate of approximately $20^{\circ} \mathrm{C}$ per minute. As the temperature approached $550^{\circ} \mathrm{C}$, the sulfur slowly evaporated, and then the chamber was heated to $850^{\circ} \mathrm{C}$ at a slower pace of $\sim 5^{\circ} \mathrm{C}$ per minute. After the temperature was kept at $850{ }^{\circ} \mathrm{C}$ for $10 \mathrm{~min}$, at such a high temperature, $\mathrm{MoO}_{3}$ powder evaporated and reacts with sulfur vapor to 
form volatile suboxide $\mathrm{MoO}_{3-\mathrm{x}}{ }^{[13]}$ these suboxide compounds diffused to the substrate and further reacted with sulfur vapor to grow $\mathrm{MoS}_{2}$ films ${ }^{[15]}$. Finally, the furnace was cooled down to room temperature naturally. Ultra-high purity $\mathrm{N}_{2}$ gas was flowed with the flowing rate of $100 \mathrm{sccm}$ during the whole growth process. Optical microscopy (OM) images, Raman spectroscopic and photoluminescence (PL) spectroscopic analyses were conducted to observe the surface morphology of the films, the structural and optical property of the grown $\mathrm{MoS}_{2}$ thin films.

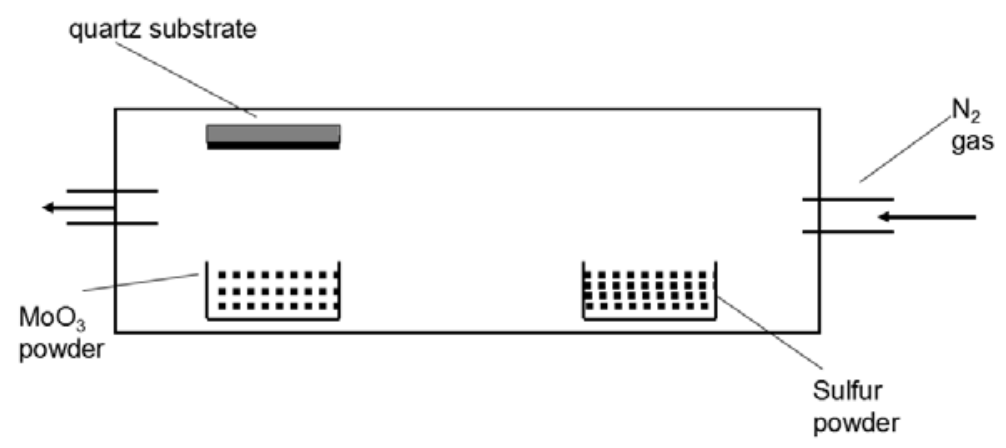

Figure 1 Schematic diagram of the CVD system used for the growth of $\mathrm{MoS}_{2}$.

It has been reported that $\mathrm{MoS}_{2}$ formed as the result of the stepwise reaction of substitution and reduction which lead to an intermediate product of $\mathrm{MoO}_{3-\mathrm{x} \cdot}{ }^{[13,16]}$ Although the growth dynamics of $\mathrm{MoO}_{3-\mathrm{x}}$ and sulfur are still not fully understood, there are two possible channels for the surface growth that can be expected: (1) $\mathrm{MoO}_{3-\mathrm{x}}$ species adsorb and diffuse on the substrate, reacting with $\mathrm{S}$ to form $\mathrm{MoS}_{2}$; (2) $\mathrm{MoO}_{3-\mathrm{x}}$ and sulfur react directly in the gas phase, and the resulting $\mathrm{MoS}_{2}$ clusters adsorb, nucleate, and grow on the substrate ${ }^{[17]}$.

In our experiment, the reaction process is described as follows. Firstly, a precursor $\mathrm{MoO}_{3}$ powder and the quartz substrate are placed in downstream location in furnace. When the temperature in the central furnace rise up to $550^{\circ} \mathrm{C}$, the $\mathrm{MoO}_{3}$ powder begins evaporating. Meanwhile, the sulfur powder also evaporates, and is conveyed by $\mathrm{N}_{2}$ gas to downstream in the furnace. The $\mathrm{MoO}_{3}$ then react with sulfur vapour to form volatile $\mathrm{MoO}_{3-\mathrm{x}}$ species, which subsequently deposit on the quartz substrate to form $\mathrm{MoO}_{3-x}$ microplates. Finally, the oxygen atoms in the well-ordered packing of $\mathrm{MoO}_{3 \text {-x }}$ are replaced with sulfur atoms to form $\mathrm{MoS}_{2}$ films on the substrate. Considering the fact that $\mathrm{MoO}_{2}$, the most common intermediate product, is one of the most stable oxides of molybdenum, we can assum the $\mathrm{x}=1$. A possible stepwise reaction process of $\mathrm{MoO}_{3}$ and $\mathrm{S}$ is given in equations (1) and $(2)^{[13]}$.

$$
\begin{gathered}
\mathrm{MoO}_{3}+\frac{1}{2} \mathrm{~S} \rightarrow \mathrm{MoO}_{2}+\frac{1}{2} \mathrm{SO}_{2} \\
\mathrm{MoO}_{2}+3 \mathrm{~S} \rightarrow \mathrm{MoS}_{2}+\mathrm{SO}_{2}
\end{gathered}
$$

\section{Results and discussion}

\section{Optical microscopy images of the $\mathrm{MoS}_{2}$ layers}

Figure 2 shows the $\mathrm{OM}$ image of the layered $\mathrm{MoS}_{2}$ films grown on quartz substrate. It indicates that we fabricated $\mathrm{MoS}_{2}$ films on the quartz substrate. The largest area of the films is about $20 \times 20 \mu \mathrm{m}^{2}$, indicating uniform $\mathrm{MoS}_{2}$ film. Furthermore, as the optical image shows, the shape of the $\mathrm{MoS}_{2}$ is rhombus. This can be explained that the intermediate product $\mathrm{MoO}_{2}$ films was nucleated on quartz substrates and grew up to rhomboidal microplates, which is in consistent with the work of Xinsheng Wang etc ${ }^{[18]}$. They thermally evaporated $\mathrm{MoO}_{2}$ powder on the $\mathrm{SiO}_{2} / \mathrm{Si}$ substrate, and it grew up to rhomboidal microplates. D. O. Scanlon etc. ${ }^{[19]}$ theoretically proved that the shape of $\mathrm{MoO}_{2}$ films to be closely related to rutile, which further confirms our assumption. 


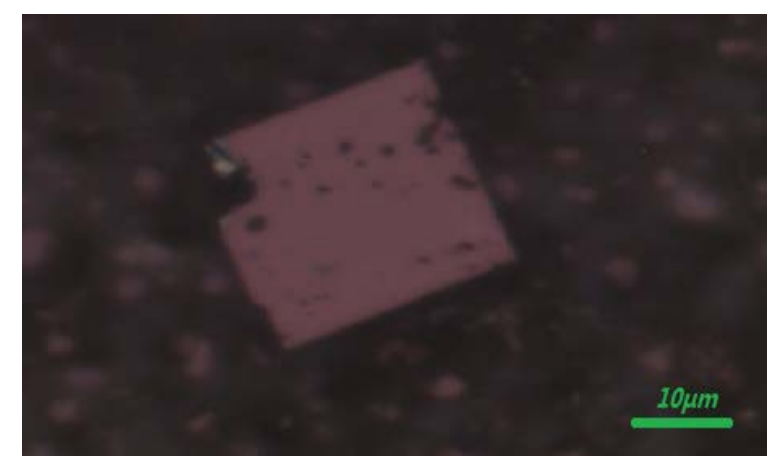

Figure $2 \mathrm{OM}$ images of the layered $\mathrm{MoS}_{2}$ films grown on quartz substrate.

\section{Raman spectra}

Figure 3 shows the Raman spectra of the monolayer $\mathrm{MoS}_{2}$ and the inset shows the bulk $\mathrm{MoS}_{2}$ film in other area of the quartz substrate. Both Raman spectras of the monolayer $\mathrm{MoS}_{2}$ films show two signature peaks, which are in-plane and perpendicular-to-plane Raman active modes respectively. In $\mathrm{E}_{2 \mathrm{~g}}{ }^{1}$ mode both $\mathrm{Mo}$ and $\mathrm{S}$ atoms vibrate along the in-plane direction (yet opposite to each other), whereas in the $\mathrm{A}_{1 \mathrm{~g}}$ mode the $\mathrm{S}$ atoms vibrate in the perpendicular-to-plane direction ${ }^{[20]}$. Raman spectroscopy could serve as a reliable tool for identifying monolayer $\mathrm{MoS}_{2}$, and the $\mathrm{E}_{2 \mathrm{~g}}{ }^{1}$ and $\mathrm{A}_{1 \mathrm{~g}}$ peak frequencies can be used to identify the layer number of an ultrathin $\mathrm{MoS}_{2}$ flake with high accuracy ${ }^{[21]}$. Figure 3 exhibits two Raman characteristic peaks at $405 \mathrm{~cm}^{-1}\left(\mathrm{E}_{2 \mathrm{~g}}{ }^{1}\right)$ and $386 \mathrm{~cm}^{-1}\left(\mathrm{~A}_{1 \mathrm{~g}}\right)$ where the distance between the in-plane $E_{2 \mathrm{~g}}{ }^{1}$ mode and out-of-plane $A_{1 \mathrm{~g}}$ mode is $19 \mathrm{~cm}^{-1}$, indicating the formation of monolayer $\mathrm{MoS}_{2}$, consistent with other reports ${ }^{[21,22]}$. As a comparison, for the bulk $\mathrm{MoS}_{2}$ films the Raman shift between the $\mathrm{E}_{2 \mathrm{~g}}{ }^{1}$ mode and $\mathrm{A}_{1 \mathrm{~g}}$ mode is $25 \mathrm{~cm}^{-1}$. In comparison, we also found in some areas of the grown film is bulk $\mathrm{MoS}_{2}$ film, the Raman characteristic peaks of which are $384 \mathrm{~cm}^{-1}\left(\mathrm{E}_{2 \mathrm{~g}}{ }^{1}\right)$ and $409 \mathrm{~cm}^{-1}\left(\mathrm{~A}_{1 \mathrm{~g}}\right)$.

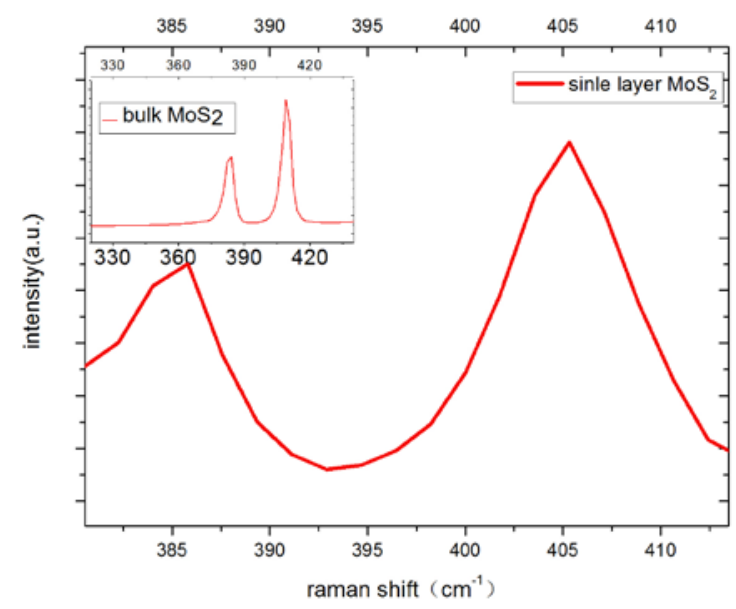

Figure 3 Raman spectra of monolayer $\mathrm{MoS}_{2}$ inset shows the Raman spectra of bulk $\mathrm{MoS}_{2}$ film in other area of the quartz substrate.

\section{PL spectrum}

The presence of monolayers is then confirmed by performing PL measurements. The PL of monolayer $\mathrm{MoS}_{2}$ was measured at room temperature using the $532 \mathrm{~nm}$ laser (Figure 4). A strong photoluminescence is observed at the direct excitonic transitions energies in a monolayer $\mathrm{MoS}_{2}$. The PL spectrum of monolayer $\mathrm{MoS}_{2}$ exhibits two peaks at $670 \mathrm{~nm}(1.85 \mathrm{eV})$ and $620 \mathrm{~nm}(2 \mathrm{eV})$. They are associated with excitonic transitions at the $\mathrm{K}$ point of the Brillouin zone ${ }^{[6]}$, which can be correlated to the A1 excitation and B1 excitation of $\mathrm{MoS}_{2}$, respectively. The dominated PL peak at $670 \mathrm{~nm}$ arises from the direct intraband recombination of the photogenerated electron-hole pairs in 
the monolayer layer $\mathrm{MoS}_{2}$, and the weak shoulder peak at $620 \mathrm{~nm}$ is attributed to the energy split of valence band spinorbital coupling of $\mathrm{MoS}_{2}{ }^{[6,23]}$. The energy difference of $0.15 \mathrm{eV}$ in PL spectrum is in good agreement with the theoretical value of $0.148 \mathrm{eV}$, calculated for monolayer $\mathrm{MoS}_{2} \cdot{ }^{\left[{ }^{23]}\right.}$ Such luminescence is absent in the indirect bandgap bulk $\mathrm{MoS}_{2}$ films. This observation is consistent with the theoretical prediction of indirect to direct bandgap transition with the structure of $\mathrm{MoS}_{2}$ turning from multilayer to monolayer.

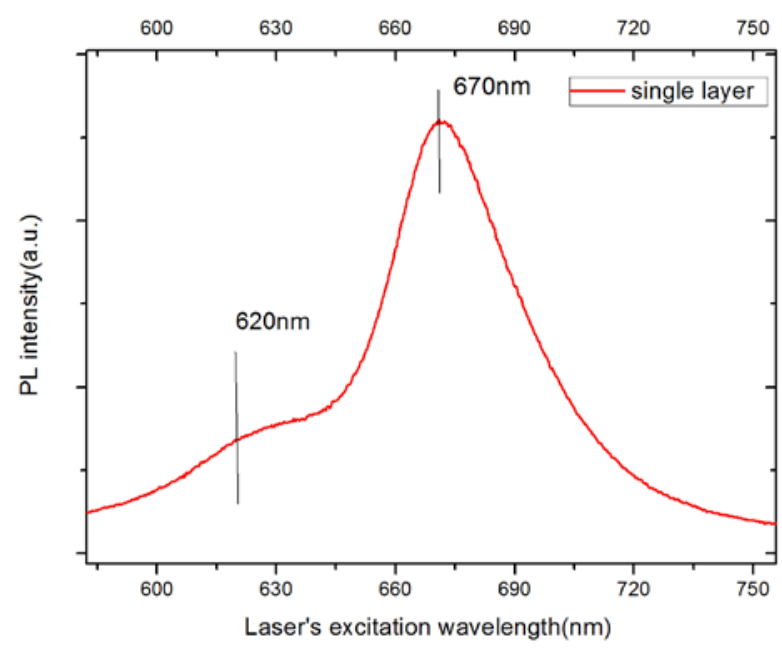

Figure 4 Photoluminescence spectra of monolayer $\mathrm{MoS}_{2}$. The laser excitation wavelength is 532 nm.

\section{Conclusions}

In summary, we fabricated the monolayer $\mathrm{MoS}_{2}$ directly on quartz substrate by CVD approach. The OM image shows we fabricated $\mathrm{MoS}_{2}$ films on the quartz substrate. The largest area of the films is about $20 \times 20 \mu \mathrm{m}^{2}$, indicating uniform $\mathrm{MoS}_{2}$ film. The Raman spectra and PL spectrum co-prove that we fabricated monolayer $\mathrm{MoS}_{2}$ film on the quartz substrate. The Raman shift of characteristic peaks of monolayer $\mathrm{MoS}_{2}$ is $19 \mathrm{~cm}^{-1}$. Whereas in bulk MoS films, the Raman shift of characteristic peaks between the $\mathrm{E}_{2 \mathrm{~g}}{ }^{1}$ mode and $\mathrm{A}_{1 \mathrm{~g}}$ mode is $25 \mathrm{~cm}^{-1}$. In PL spectrum, we find a strong photoluminescence at $670 \mathrm{~nm}$ in a monolayer $\mathrm{MoS}_{2}$. This suggests that monolayer $\mathrm{MoS}_{2}$ is direct bandgap semiconductor, the optical bandgap of which turns to be $1.85 \mathrm{eV}$. Besides, the photoluminescence is absent in the indirect bandgap bulk $\mathrm{MoS}_{2}$ films. This work opens an avenue to develop single-layer semiconducting materials for future photoelectric device applications in switches, memories, signal-amplifiers, and light-related sensors, photovoltaic etc.

\section{Acknowledgements}

This work is supported by National Natural Science Foundation of China (Grant No.51472096). We also acknowledge the Analytical and Testing Center of Huazhong University of Science and Technology for Raman spectra and PL spectrum measurements.

\section{Reference}

[1]R. Ganatra, Q. Zhang: 'Few-layer MoS2: ‘a promising layered semiconductor”, ACS nano, 2014, 8(5): 4074-4099.

[2]B. Radisavljevic, A. Radenovic, J. Brivio: 'Single-layer MoS2 transistors', Nature nanotechnology, 2011, 6(3): 147-150. 
[3]J. Pu, Y. Yomogida, K. K. Liu,: 'Highly flexible MoS2 thin-film transistors with ion gel dielectrics', Nano letters, 2012, 12(8): 4013-4017.

[4]T. Korn, S. Heydrich , M. Hirmer: 'Low-temperature photocarrier dynamics in monolayer MoS2’, Applied Physics Letters, 2011, 99(10): 102109.

[5]K. F. Mak, C. Lee, J. Hone: ‘Atomically thin MoS 2: a new direct-gap semiconductor’ Physical Review Letters, 2010, 105(13): 136805.

[6]A. Splendiani, L. Sun, Y. Zhang: 'Emerging photoluminescence in monolayer MoS2', Nano letters, 2010, 10(4): 1271-1275.

[7]R. Fivaz, E. Mooser: 'Mobility of charge carriers in semiconducting layer structures' Physical Review, 1967, 163(3): 743.

[8]H. Wang, L Yu, Y. H. Lee: 'Integrated circuits based on bilayer MoS2 transistors', Nano letters, 2012, 12(9): 4674-4680.

[9]A. Ayari, E. Cobas, O. Ogundadegbe: 'Realization and electrical characterization of ultrathin crystals of layered transition-metal dichalcogenides', Journal of applied physics, 2007, 101(1): 14507-14507.

[10]J. N. Coleman, M. Lotya: 'Two-dimensional nanosheets produced by liquid exfoliation of layered materials’, Science, 2011, 331: 568-571.

[11]P. Joensen, R. F. Frindt, S. R. Morrison: 'Single-layer MoS2', Mater Res Bull, 1986, 21: 457-461.

[12]K. Ellmer: 'Preparation routes based on magnetron sputtering for tungsten disulfide (WS2) films for thin

-fЙ60.solar cells', Physica status so

[13]X. L. Li, Y. D. Li: 'Formation of MoS2 inorganic fullerenes (IFs) by the reaction of MoO3 nanobelts and S’, Chemistry-A European Journal, 2003, 9(12): 2726-2731.

[14]S. Balendhran, J. Z. Ou, M. Bhaskaran: 'Atomically thin layers of MoS 2 via a two step thermal evaporation-exfoliation method’, Nanoscale, 2012, 4(2): 461-466.

[15]Y. H. Lee, X. Q. Zhang, W. Zhang: 'Synthesis of Large

Chemical Vapor Deposition’, Advanced Materials, 2012, 24(17): 2320-2325.

[16]T. Weber, J. C. Muijsers: 'facial method to prepare MoS2 with nanoflower-like morphology', Phys. Chem. 1996, 100, $14144-14150$

[17]Q. Ji, Y. Zhang: 'Chemical vapour deposition of group-VIB metal dichalcogenide monolayers: engineered substrates from amorphous to single crystalline’, Chemical Society Reviews, 2015.

[18]X. Wang, H. Feng, Y. Wu: 'Controlled synthesis of highly crystalline MoS2 flakes by chemical vapor deposition’, Journal of the American Chemical Society, 2013, 135(14): 5304-5307.

[19]D. O. Scanlon, G. W. Watson, D. J. Payne: 'Theoretical and experimental study of the electronic structures of MoO3 and MoO2', The Journal of Physical Chemistry C, 2010, 114(10): 4636-4645.

[20]M. Shanmugam, C. A. Durcan, B. Yu: 'Layered semiconductor molybdenum disulfide nanomembrane based Schottky-barrier solar cells’, Nanoscale, 2012, 4(23): 7399-7405.

[21]H. Li, Q. Zhang, C. C. R. Yap: 'From bulk to monolayer MoS2: evolution of Raman scattering', Advanced Functional Materials, 2012, 22(7): 1385-1390.

[22]R. S. Sundaram, M. Engel, A. Lombardo: 'Electroluminescence in single layer MoS2' Nano letters, 2013, 13(4): 1416-1421.

[23]Z. Y. Zhu, Y. C. Cheng: 'Schwingenschlögl U. Giant spin-orbit-induced spin splitting in 
two-dimensional transition-metal dichalcogenide semiconductors', Physical Review B, 2011, 84(15): 153402. 